\title{
Recent results of CHIMERA activity
}

\author{
A. Pagano
}

INF, Sezione di Catania, Italy

\begin{abstract}
The experimental activity of CHIMERA in recent years has been characterized by a steady progress in the detection technique and data analysis. Since 2008 the detector system benefits of new implementations: a new reaction chamber, a new charged particle identification in silicon detector made by an extended pulse shape method and an efficient system for the identification of exotic beams produced by projectile-like fragmentation (In-flight method). These implementations appear to be promising tools in view of further exclusive experiments in the field of isospin physics. The coupling of CHIMERA with other equipments (such as interferometers and highly segmented arrays, magnetic elements, neutron detectors, etc.) is also envisaged in order to extend the studies of the reaction mechanism in heavy ion physics.
\end{abstract}

\section{Introduction}

The aim of this brief report is to illustrate the recent activity of CHIMERA in the physics of heavy ions collisions at Fermi energy. Chimera activity started by phase one, with the REVERSE campaign on 1999 at Laboratori Nazionali del Sud in Catania (LNS) by using ${ }^{124,112}$ Sn projectiles at 25 and $35 \mathrm{MeV} /$ nucleon. At that time only the FORWARD part of CHIMERA was available. Experiments in reverse kinematics were started by using nuclear targets of Nickel 58 and 64 isotopes. Since then, the detector was running successfully in different experiments and, on 2003 the whole apparatus was ready for the full $4 \pi$ detection, that was accomplished in the large 
reaction chamber CICLOPE at LNS. During years 2002-2008 huge efforts were done to increase the detection capability toward a more complete ion identification at low kinetic energy in such a way to identify in charge light ions stopped in the first stage of the large-surface silicon detectors of the CHIMERA telescopes. The goal was accomplished in 2008 by introducing an efficient low threshold pulse shape identification (PSD) method in silicon. In the mean time, a new dedicated beam line equipped to allocate a new reaction chamber was ready at LNS. The whole detector made by $1192 \mathrm{Si}-\mathrm{CsI}(\mathrm{Tl})$ telescopes was then installed in the new reaction chamber CHIMERA. Recently, a brief report, comprehensive of a short history of the detector and almost a complete lists of basic papers describing the detection system has been published [1]. In this IWM report, the attention is focused about more recent achievements, essentially starting from 2008 with emphasis on the possibility of isospin physics studied with stable and exotic beams. Indeed, future programs with the CHIMERA detector will devote much attention to exotic nuclei, exotic decays and high resolution correlation measurements, also performed with dedicated coupling of new devices, such as the FARCOS array [2].

\section{Recent progresses in experimental methods with CHIMERA}

\section{$2.1 \quad$ Identification methods}

An extensive study of the shape of signals produced in silicon and $\mathrm{CsI}(\mathrm{Tl})$ detectors performed by both conventional and digital pulse shape acquisition was undertaken by CHIMERA working group [3]. One of the main goals of this study consisted in further advancing towards the renewed interest in charged particle discrimination with (PSD) method. So, after some studies, an upgrade of the CHIMERA detector has been envisaged [4]. In this way, mass (by Time of flight) and charge (by PSD) identifications for reaction products stopping in silicon detector were achieved. A recent experimental campaign (INFN/EXOCHIM experiment) has been started on January 2008 at LNS, incorporating a new electronics front-end that allows charge identification of slowly moving light fragments $(\mathrm{Z}<15$ atomic charge units) by means of the PSD technique.

It is important to notice that good results in charge identification with silicon detectors were reported in the literature by using relatively low capacitance prototypes silicon n-type detector of low resistivity, operating in 


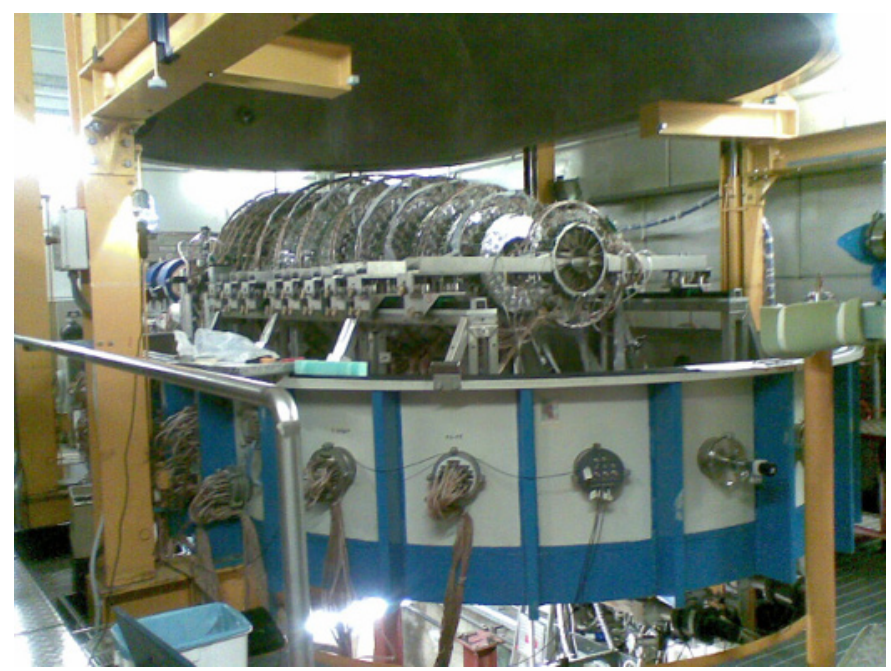

Figure 1: Recent (2008) installation of CHIMERA detector at LNS.

REVERSE mode. Identification threshold as lower as 1-2 MeV/nucleon have been reported [5]. In CHIMERA the experimental conditions were almost different with respect to Ref. [5]: The Time of Flight Technique(TOF), used for mass identification, the large surface silicon detectors of moderately high resistivity already adopted ( to reduce detector bias) suggested to employ the pulse shape methods by using "CHIMERA standard" n-type planar detectors operating in DIRECT mode. After some studies, it was demonstrated that a reasonable low identification threshold as low as $4.5 \mathrm{MeV} /$ nucleon for light ions was possible by adopting an appropriate rise time measurement of the silicon charge signal [6]. This latter study has triggered the experiment to perform an adaptation of the front-end electronics in such a way to incorporate the rise time measurement(PSD) in addition to the TOF method. Beside PSD charge discrimination, such an extension allows for additional information and quality of the results in view of an off-line correction of the time $t_{0}$ delay ( energy dependent for each individulal detector ) in the stop signals of the 1200 silicon detectors employed by CHIMERA with respect to the RF common start signal delivered by the Super conductive cyclotron (SCC). 

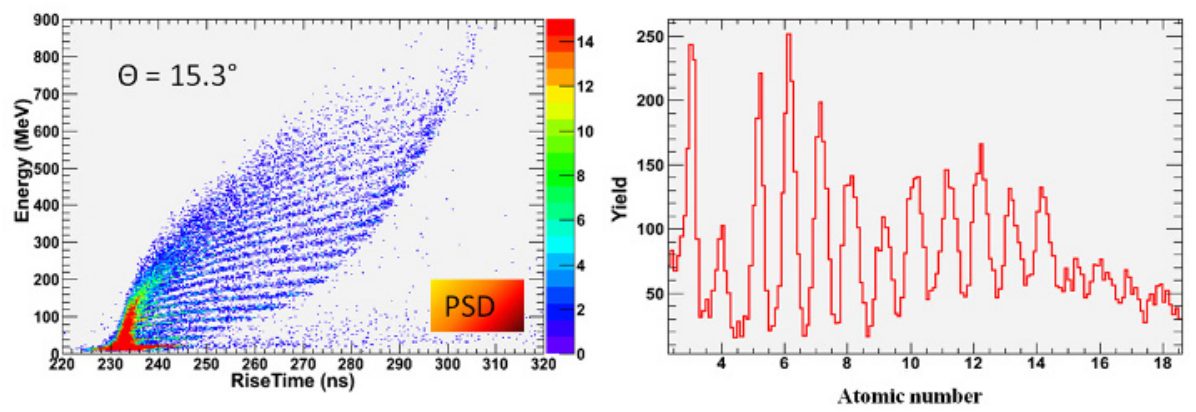

Figure 2: Pulse shape identification: charge of light fragments stopped in the CHIMERA silicon detector.Nuclear reaction: ${ }^{64} \mathrm{Ni}+{ }^{124} \mathrm{Sn}$ at $35 \mathrm{~A} . \mathrm{MeV}$. Left side: Kinetic energy vs rise-time. Right side: Projection of the charge identification after PSD linearization.

\subsection{Time of flight system for exotic beam produced by projectile-like fragmentation}

The new CHIMERA beam line was also equipped with an efficient MicroChannel Plate timing system and a microstrip silicon tagging detector [7], in order to identify, by means of E-TOF techniques, exotic beams produced by projectile fragmentation of primary beams delivered by the LNS-SCC accelerator [8]. This latter characterization is an important aspect of the present scientific programs of CHIMERA, mainly devoted to the study of nuclei with large isospin asymmetry, which is expected to determine the evolution of the reaction mechanism for both binary, compound nucleus reactions and multifragmentation [9]. Different tests have been accomplished during the last two years with important advancements in both the identification of light and medium mass exotic beams up to atomic numbers close to $\mathrm{Zn}$ element. The limitation in obtaining beam intensities larger than $10^{3}-10^{4}$ particle/s (for a favorable neutron to proton ratio) is due mainly to the limitation in the power dissipation of the primary beam at the surface of the main deflector system just at the extraction point of the SCC accelerator. Indeed, simulations and experimental tests indicate a transmission beam line of high efficiency [10]. Recently, it has been proposed [11] to use a ${ }^{70} \mathrm{Zn}$ (40 A.MeV) as primary beam in order to produce neutron rich Nickel isotopes. In a recent test, a ${ }^{70} \mathrm{Zn}^{19+}(40 \mathrm{~A} . \mathrm{MeV})$ primary beam impinging on a $250 \mu \mathrm{m}$ ${ }^{9}$ Be target has been used.The maximum intensity obtained for the primary beam was about $300 \mathrm{enA}(0.03 \mathrm{~kW})$. The magnetic settings of the fragment separator were optimized for the transport of the ${ }^{68} \mathrm{Ni}(\mathrm{B}=2.0335 \mathrm{Tm})$. Im- 


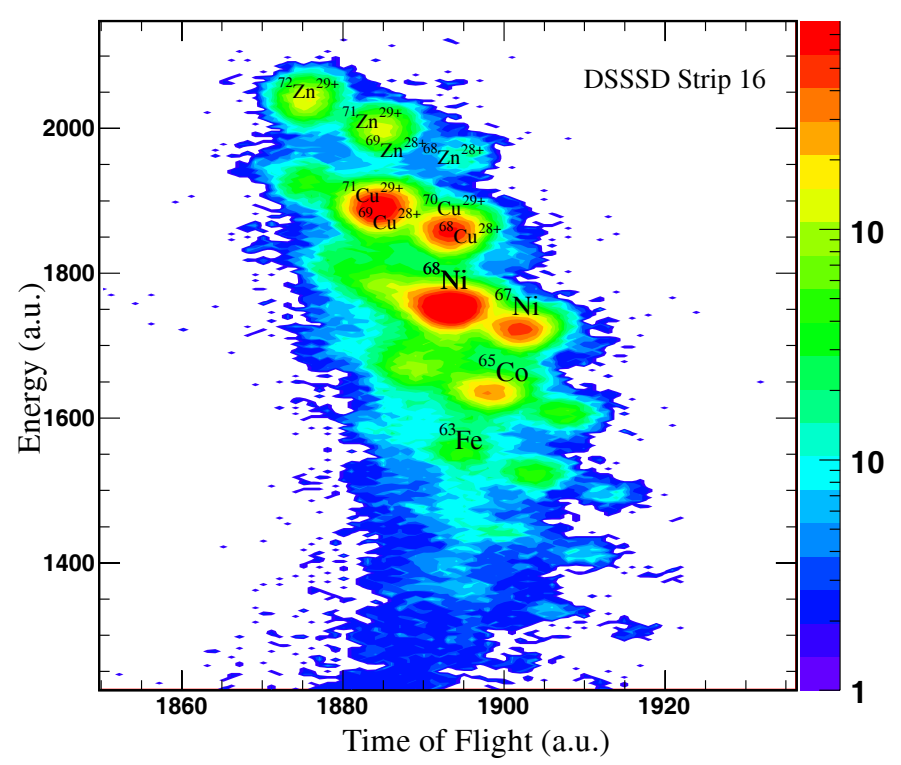

Figure 3: Identification Energy-time of flight array of $\mathrm{Ni}$ isotopes obtained by In-Flight Fragmentation method. The identification system of exotic beam was developed in order to fit with experimental characteristics of CHIMERA beam line.

proved transportation was obtained using a plastic scintillators diagnostic points along the beam line. Identification of different beams was obtained using the CHIMERA-IFEB tagging system equipped with a Micro Channel Plate (MCP) [7], and a Double Side Silicon Strip Detector (DSSSD). The two detectors, DSSSD and MCP, give the energy loss and the time-of-flight measurements ( respect to MCP and with flight path of $13 \mathrm{~m}$ ) allowing the event-by-event identification of the incoming beams. We used a $32 \times 32$ strips, 6x6 $\mathrm{mm} 2$ surface, $140 \mu \mathrm{m}$ thick DSSSD, similar to the ones adopted by the FARCOS detector prototypes [2]. A final energy of $28 \mathrm{~A} . \mathrm{MeV}$, in the case of ${ }^{68} \mathrm{Ni}$ isotope after transmission through the tagging system, was achieved. Fig. 3 shows the obtained pattern identification for $\mathrm{Ni}$ isotopes and other elements of different isotopes.

\subsection{Symmetry energy at supra saturation density}

The study of the symmetry energy at nuclear densities up to 2-3 times the saturation density $\left(\approx 0.17\right.$ nucleon $\left./ \mathrm{fm}^{3}\right)$ also attracts large interest by the 
heavy-ion physics and astrophysics communities [12]. The unique features of CHIMERA, as a powerful selector and a quasi-ideal filter for different class of events, continue to stimulate considerable efforts and projects in heavy ion physics for more exclusive measurements. In the context of the ASY-EOS Collaboration, an experimental investigation at GSI has been accomplished in order to study ${ }^{197} \mathrm{Au}+{ }^{197} \mathrm{Au},{ }^{96} \mathrm{Ru}+{ }^{96} \mathrm{Ru}$ and ${ }^{96} \mathrm{Zr}+{ }^{96} \mathrm{Zr}$ collisions at 400 $\mathrm{MeV} /$ nucleon. In this experiment the forward part of the CHIMERA device has been successfully coupled to the Land neutron detector, the Aladin ToF-Wall, the Si-CsI Krakow array, and the CsI(Tl) Washington University Microball detector with the aim of studying neutron and proton elliptic flows that are expected to be very sensitive to the stiffness of symmetry energy of the nuclear equation of state at supra-saturation densities [13].

\subsection{Detection developments-new proposals}

The fact that silicon detectors are largely employed in CHIMERA suggested to study the possibility to measure neutron in direct two/three body reactions under suitable kinematical coincidence condition [14]. With this method it is also possible to employ the whole CHIMERA apparatus in specific experimental situations, in connection with exotic beams delivered by EXCYT facility or by In-Flight fragmentation method. However, it is well known that neutron detection in a $4 \pi$ configuration is only partially accomplished by dedicated neutron devices and no full integration of neutron detection with charged particle spectroscopy has been achieved till now [15]. In a $4 \pi$ detector like CHIMERA, employing so large number of silicon detectors, one is naturally draw to think about the possibility to detect neutron by a recoil proton (or light charged particle) method already known since early assessments of experimental methods in nuclear physics [16].

Indeed, one important progress of CHIMERA detection is represented by the possibility to couple it with advanced detectors in order to have access to specific portion of the available phase space. Correlations methods have been carefully studied with CHIMERA by running a first generation experiment (BOSON CONDENSATION) aimed to reconstruct ${ }^{12} \mathrm{C}$ excited nuclei produced in heavy ion collisions by detecting three alpha particles in the final state and using CHIMERA as main correlator [17]. Correlation experiments open new frontiers in the accessible physics that can be studied with CHIMERA or third generation devises [18]. However, in order to progress in this research line it was recently suggested to accept only proposals in CHIMERA that will include in the detection system dedicated advanced devices in order to improve the angular resolutions in some well 
defined portions of the available phase space.

Neutron detection with CHIMERA is under consideration and explorative studies have been undertaken by benefiting also of important efforts made by European collaborations in nuclear spectroscopy and neutron detection advancements (such as, for example NEDA collaboration). It is evident that the present location of the CHIMERA reaction chamber and its heavy frame mechanical structure, are not favorable experimental conditions for a proper neutron detection system; so, different modifications in the apparatus have to be envisaged in order to allow for neutron detection. Unfortunately, it happens that in recent time, the consistent reduction of budget allocated for human resources, the extreme difficulty to give to young generation a reasonable perspective for future employments introduce important constraints for future developments and progresses in the detection technique. However, this latter problem is not a peculiar problem of CHIMERA group. Indeed, it represents a dramatic tendency around the world, now day.

\section{Recent progress in data analysis}

Processes of statistical and dynamical multifragmentation have been investigated in a broad range of projectile-target combinations at Fermi energies, with particular emphasis on the time scale of the reaction dynamics. These studies were accomplished by profiting from the capabilities of the detector to perform good time of flight measurements in a $4 \pi$ configuration, as well as good isotopic identifications of light fragments, mostly in the forward direction. The reaction dynamics and the isospin degree of freedom have been investigated in specific nuclear reactions such as neck fragmentation and isospin diffusion, compound nucleus formation and decay, fission and exotic decay of very heavy nuclear systems [19]. It is not possible in this brief report to summarize all the results obtained during the different phases of the CHIMERA projects; so, only few recent studies undertaken with CHIMERA have been shortly presented; some reports are published in these IWM proceedings. In this short report only progresses in the field of TIME SCALE analysis will be discussed.

The capability of CHIMERA to detect fragments in a broad range of kinetic energies, ranging from slow moving target-like residues (TLF) up to fast moving projectile-like residues (PLF), has been one of the most important innovations attained by CHIMERA among the $4 \pi$ detection systems [1]. This feature was successfully applied to establish a new method of investi- 
gation based on the evaluation of fragment-fragment relative velocities in a three-body kinematical analysis and coulomb trajectory simulations to calibrate the time scale of the emission of intermediate mass fragments (IMF), produced in semi-peripheral collisions. The method has also triggered theoretical studies in the field of transport theories (BNV,QMD), opening the possibility of new experimental studies of the reactions mechanism with exotic beams [20].

The time scale analysis was firstly developed in CHIMERA for three body channel in the reaction ${ }^{124} \mathrm{Sn}+{ }^{64} \mathrm{Ni}$, at $\mathrm{E}_{l a b}\left({ }^{124} \mathrm{Sn}\right) / \mathrm{A}=35$ $\mathrm{MeV} /$ nucleon. In the three body reactions, the measured velocities of the three partners, respectively: Projectile-like fragments (PLF), Targetlike fragments (TLF) and Intermediate mass fragments (IMF), were correlated by simple procedures. Basically the methods works as it follows. The experimental relative velocities of IMFs with respect to PLFs and TLFs, $\mathrm{V}_{R E L}(\mathrm{IMF}, \mathrm{PLF})$ and $\mathrm{V}_{R E L}(\mathrm{IMF}, \mathrm{TLF})$, respectively, are divided by the relative velocity $\mathrm{V}_{V I O L A}$, corresponding to the released kinetic energy, determined by the Coulomb repulsion for the binary splitting of the two sub-systems (IMF,PLF) and (IMF,TLF), as given by the Viola systematic. The evaluation of the time scale (sketched by a full line in the fig.4) was obtained by simulating complete collinear motion of IMFs along with the PLF-TLF relative velocity vector, in good agreement with the experimental observation. Events approaching (see fig.4)) the values: $\mathrm{V}_{R E L} / \mathrm{V}_{V I O L A}(\mathrm{IMF}, \mathrm{PLF})=1$ and $\mathrm{V}_{R E L} / \mathrm{V}_{V I O L A}(\mathrm{IMF}, \mathrm{TLF})=1$ (i.e., in agreement with the Viola systematic) correspond to sequential decay of the primary PLF nucleus or the TLF nucleus, respectively. Those events that are close to the first diagonal in the plot of fig.4), are not consistent with a pure coulomb binary repulsion as predicted by the systematic. These events were interpreted by prompt ternary divisions. The localization of the events in fig.4), clearly demonstrates that light IMFs (most probable) are emitted in almost prompt or "fast two-step" processes.

The method was also important in establishing an important hierarchy effect in the emission time of IMFs. In fact, it was observed that with increasing IMF's charge the corresponding emission time scale also increases to values as large as $300 \mathrm{fm} / \mathrm{c}$ or more, typical for those IMFs originating from nearly sequential asymmetric binary fission of a primary PLF into two big massive fragments [21]. Time scale vs. Isospin dynamics with CHIMERA was also studied [22], in order to probe the concept of isospin diffusionand isospin drift as a function of the density gradient. It was found that fragments dynamically emitted from the early stage of the reaction show larger values of the isospin asymmetry (N-Z/A), thus indicating a favored 


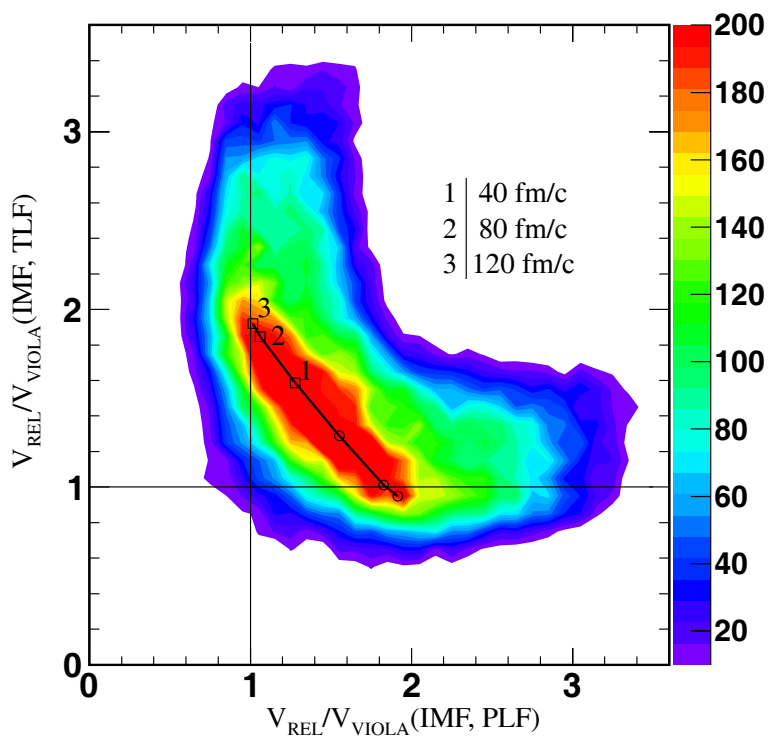

Figure 4: Illustration of the time scale method performed with CHIMERA detector (adapted from ref. [1]). The Correlation plot between relative velocities of the sub-systems (IMF,PLF) and (IMF,TLF) is obtained for different IMF with atomic numbers, $4 \leq Z_{I M F} \leq 10$. The experimental distributions are compared with simple simulations assuming that IMFs are released as a result of a two-step collinear neck rupture process taking place 40,80 , or $120 \mathrm{fm} / \mathrm{c}$ after re-separation of the primary interacting projectile-target binary system.

emission of more neutron-rich nuclei as compared to fragments sequentially emitted in a later stage of the reaction mostly from splitting of TLF and PLF partners.

Time scale investigations in collisions of very heavy non-fusing nuclear systems were also investigated at different bombarding energies. A recent study of the collision ${ }^{197} \mathrm{Au}+{ }^{197} \mathrm{Au}$ at energies of $15 \mathrm{MeV} /$ nucleon, revealed unexpected fast re-separation of heavy nuclei into three or four fragments of comparable size [23], opening a novel understanding in the transition mechanism from deep inelastic collisions to multifragmentation processes in heavy-ion systems. The physics case, briefly discussed in this section, shows that the time scale evaluation of nuclear reactions is an essential information in order to probe the isospin dynamics and the reaction mechanism in heavy ion collisions. 


\section{Summary and Outlook}

After ten years of experimental activity, CHIMERA is a lively $4 \pi$ detector well suited to nuclear reaction studies around Fermi energies with stable and exotic beams. The experimental activity has been evolving through different phases, always characterized by a steady progress in the detection technique and data analysis. Further experimental progresses towards a better isotopic identification of charged particles and the possibility to include neutron detections appear to be promising perspectives in view of extensive experiments in the fields of exotic nuclei and isospin physics. The coupling of CHIMERA to other equipments (such as interferometers and highly segmented arrays, magnetic elements, neutron detectors, etc.) is strongly encouraged in order to expand our exploration and understanding of specific features in the reaction mechanism.

\section{References}

[1] A. Pagano, Nuclear Physics News International, 22, No.1, 28-33 (2012).

[2] L. Acosta, E.V. Pagano et al., see IWM2011 conference proceedings in this volume.

[3] P. Guazzoni, et al, Proceedings of IWM2005, Italian Physical Society, Ed. R. Bougault, A. Pagano, S.Pirrone, M-F. Rivet and F.Rizzo, Catania, 28 November-1 December, Vol. 91, p. 51 (2005); M. Alderighi, et al., IEEE Trans. on Nuclear Science, 53, No.1, 279 (2006); M. Alderighi et al., Nucl. Phys. A734 Supplement, E88-E91 (2004); F. Amorini et al., IEEE Trans. on Nuclear Science 54, 208 (2007).

[4] R. Bassini, C. Boiano, A. Pagano, et al., Nucl. Science Symposium Conf. Record, 2006, IEEE Trans. on Nucl. Sci., Vol. 1, 507 (2006).

[5] M. Mutterer et al., IEEE Trans. on Nucl. Sci. 47, 756 (2000).

[6] M. Alderighi, et al., IEEE Trans. on Nucl. Sci. 52 (2005) 1624.

[7] I. Lombardo et al, Nuclear Physics B (Proc. Suppl.) 215, 272 (2011); L. Grassi, et al, LNS Activity Report ISSN 1837, 1561, p. 118 (2008).

[8] E. Rapisarda et al., Eur. Phys. J. , Special Topics 150, 269 (2007).

[9] F. Amorini et al., Phys. Rev. Lett. 102112701 (2009);S.Pirrone et al. 5th Int. Conf. FUSION11, Saint Malo, EPJ Web Conf. 17,16010 (2011). 
[10] G. Cardella et al., see proceedings in this conference.

[11] E.De Filippo et al., TIME SCALE -LNS PAC letter of intent (2012).

[12] Y.Leifels, see proceedings in this conference; R.Lemmon see proceedings in this conference.

[13] P. Russotto et al., see proceedings in this conference; W. Trautmann et al., Progr. Part. Nucl. Phys., 62425 (2009); P. Russotto, et al., Phys. Lett. B 697, 471 (2011); J. Lukasik et al., GSI Scientific Report 2010 p. 69;J. Lukasik et al., see proceedings in this conference.

[14] G. Cardella, et al., AIP Conf. Proc. American Institute of Physics, vol. 1165, p. 309 (2009).

[15] R.T. de Souza, N.Le Neindre, A.Pagano, and K.H. Schmidt, in "Dynamics and thermodinamics with Nuclear Degrees of Freedom", Ed. Ph. Chomaz, F.Gulminelli, W.Trautmann, and S.J. Yennello, Eur. Phys. J. A30 275 (2006).

[16] A.T.G. Ferguson, "Nuclear structure and electromagnetic interactions", Scottish Universities' Summer School, Ed. by N. MacDoanald, printed Oliver and Boyd, Edinburgh and London 375 (1964).

[17] B. Borderie et al., Phys. Lett. B705, 65 (2011); A. Raduta et al., see proceedings in this conference.

[18] G.Verde, see proceedings in this conference.

[19] http://www.lns.infn.it/CHIMERA; I. Lombardo et al.,see proceedings in this conference and references therein; M. La Commara et al., see proceedings in this conferences; Z.Y. Sun et al., Phys. Rev. C 82 (2010) 051603; G.Casini et al. Submitted to Phy. Rev.C (2012).

[20] V. Baran, M. Colonna, and M. Di Toro, Nucl. Phys. A730, 329 (2004); M. Papa, et al., Phys. Rev. C75, 054616 (2007); M. Di Toro, et al., J. of Phys. G: Nuclear and Particle Physics, 37, 83101 (2010).

[21] P. Russotto, et al.,Phys. Rev. C 81, (2010) 064605.

[22] E.De Filippo et al. submitted to Phys. Rev. Lett. (2012).

[23] I. Skwira-Chalot, K. Siwek-Wilczynska, J. Wilczynski, et al., Phys. Rev. Lett. 101262701 (2008); J. Wilczynski, I. Skwira-Chalot, K. SiwekWilczynska, A. Pagano, et al., Phys. Rev. C81, 024605 (2010). 